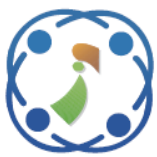

\title{
Medical Images Enhanced by Using Fuzzy Logic Depending on Contrast Stretch Membership Function
}

\author{
Rafid Abbas Ali $^{1} \quad$ Asaad M. Abbas $^{1} \quad$ Hazim G. Daway ${ }^{1 *}$ \\ ${ }^{1}$ Department of Physics, College of Science, Mustansiriyah University, Iraq \\ * Corresponding author's Email: hazimdo@uomustansiriyah.edu.iq
}

\begin{abstract}
Medical images are often adversely affected by a lack of clarity due to the limited representation of the color gamut. In this research, three types of medical images microscopic, magnetic resonance and x-ray images were enhanced by using a Fuzzy Logic by Stretch Membership Function (FLSMF). The Stretch Membership Function increased the dynamic range for the compounds red, green and blue in the medical images which have a few ranges. The FLSMF algorithm was compared with other methods by calculating the entropy value, wavelet quality evaluator and lightness order error. The analysis of the results showed that the proposed method succeeded in enhancing the contrast of the different types of medical images where it had high average values for the entropy (6.95) and wavelet quality evaluator (0.08), and a small average value for the lightness order error (60.77).
\end{abstract}

Keywords: Contrast enhancement, Fuzzy log, Lightness enhancement, Medical images, Microscopic, Magnetic resonance, X-ray.

\section{Introduction}

In recent years, Medical or microscopic images play an important part in medical image diagnosis. There are different types of medical imaging such as magnetic resonance imaging (MRI), Computerized Tomography, X-ray imaging, and microscope images. From these images, we can identify and distinguish the number of diseases, although many of these images do not have high resolution and good clarity due to the representation of limited intensity. The point of medical image improvement is to enhance the lightness and contrast in the adjacent regions to support activities such as surgical planning, monitoring, and disease diagnosis. Contrast is the distinction between bright and dark areas within the image. So, the lighting condition plays a vital role in object details appear clearly to be recognized and counted. These details are shadows and bright lights that will facilitate produce a smart distinction within the image. To improve the image brightness, it is necessary to optimize its contrast and change the details and information of the Microscopic image enhancement plays an important role in medical imagery. Some techniques include improving lightness [1, 2], and some rely on color restoration [3]. In general, some enhancement techniques use nonlinear mapping to improve lighting $[4,5]$. Others rely on the development of the technique of the Histogram Equalization (HE) [6]. Human perception is more responsive to contrast than absolute luminance [7]. Medical imaging types as magnetic resonance imaging (MRI) and Computerized Tomography (CT) and X-ray imaging generate the direct view and clear of the pathological regions. They are basic tools for detecting or diagnosing various diseases. However, there is limited imaging in the medical images due to low contrast and resolution. Medical image improvement aims to enhance the medical images in contrast or lightness to detect certain features. Some of the enhancement fuzzy techniques as in [8] used to improve color images captured at low lighting levels by using sigmoid membership function. These techniques are not suitable for medical images because most medical images suffer from lack of contrast due to low dynamic range in the red, green and blue channels and [9] presented an idea about 
using a fuzzy technique for color image captured at night. The technology improves night images by increasing the brightness of dark areas and reducing lighting in bright areas. The disadvantage of this enhancement technique is focused on improving the level of contrast for this type of images and it is not suitable. The researchers presented the night mode algorithm for different image capture devices. Preethi S.J et al. [10] introduced a membership functions ramp used to enhance the visual appearance of the image so hat maximum possible information could be extracted. They introduced two methods; Fuzzy logic Based Function Modification by Square-operation (FLFMS), and Fuzzy logic Based Function Modification by cube-operation (FLFMC) algorithms have been used to improve the usual and medical images. These methods are good at improving captured images at low and medium lighting level. However, they do not work well for images captured at low light levels, as it increases the darkness in low-lit areas. The membership function is modified for dark and bright regions but is left unchanged for the middle regions. This approach can be used in medical images to make the diagnosis easy. Raju G. et al. [11] presented a simple and effective Fuzzy color image enhancement process for enhancing color images with low contrast. This procedure is based on two parameters $\mathrm{M}$ and $\mathrm{K}$, where $M$ is average image intensity value and $K$ is a function of contrast intensification. The RGB component of the image is translated to color space HSV. The basic principle on which the technique is designed is transforming skewed histogram of the input image into a uniform histogram. The proposed algorithm is compared with conventional techniques. The proposed method is computationally faster than the existing methods and well suits with the images having a background with non-uniform distribution of brightness. Jaspreet Kaur and Amandeep Kaur in [12] Proposed Blurred histogram equalization FHE retains the brightness of the image along with enhancement of the original image in local comparison. First, Fuzzy histogram is generated from the logic of Fuzzy. Second, the histogram of Fuzzy is split down into two sub- depending on the median value or the picture data. Thereafter, each histogram is independently equalized to maximize the contrast of the image. At last, evenly distributed histograms are being combined together to get the response. Fuzzy histogram equalization involves of following important steps: -If colour picture transforms RGB to HSV. Then only add the further steps to component $\mathrm{V}$ and finally marge all components including the improved components to getting the image of enhanced. Amita Nandal and Hamurabi Gamboa
Rosales in [13] use fuzzy data to help deal with the inaccuracy of grey level values compared with conventional flat histogram-based approaches. This approach essentially prevents the presence of pictures blurred out. This is ideally suited for hilly area pictures which are suffering from poor contrast and look blurred out. The sub-blocks together are then transformed into original size blocks using inverseFTR. These inversely modified blocks are combined to reconstruct the final fused picture by choosing the most extreme dependent combination law. The suggested approach the fused image includes more detailed details and characteristics than most fused images. Denys Mikhov et al. Suggested a Fuzzylogic Technique used to enhance lightness in the colour images. A suggested number of membership functions are Gamma-Correction, white pixel and grey pixel. The best way is by analysing the results are Fuzzy-logic Based Gamma-Correction (FLBGC) [14]. Although it improves well at low light levels, it increases the illumination in the high-light area, which distorts the images in those areas.

Some medical images have low dynamic intensity levels and the range stretches can play an important role in improving it by using a fuzzy technique, in this study, the medical images are improved using fuzzy logic based on a membership function that increases the contrast using a Stretch Membership Function.

\section{Proposed method}

The fuzzy logic depending on stretch slanderedmean membership function is used to enhance the contrast and brightness in the R, G, and $\mathrm{B}$ components. The function improves the variance by histogram extraction based on mean and standard deviation values, as illustrated by a distribution before and after processing, where the enlargement of the histogram is observed. In the membership function, the minimum and maximum of each component RGB in the image are given by [3]:

$$
\begin{aligned}
& \max c_{-} c_{n}=a v c_{n}+\mathrm{t}\left(s t d c_{n}\right) \\
& \min _{-} c_{n}=a v c_{n}-\mathrm{t}\left(s t d c_{n}\right)
\end{aligned}
$$

where $n$ Represent the $\mathrm{r}, \mathrm{g}$, and $\mathrm{b}$ channels; $a v c_{n}$ and $s t d c_{n}$ are the mean value and standard deviation in the $c_{n}$ channels. $\mathrm{t}$ is default value $(\mathrm{t}=$ 2.5); The membership function is:

$$
\mu_{n}=\frac{c_{n}-\min _{-} c_{n}}{\max _{-} c_{n}-\min _{-} c_{n}} .
$$




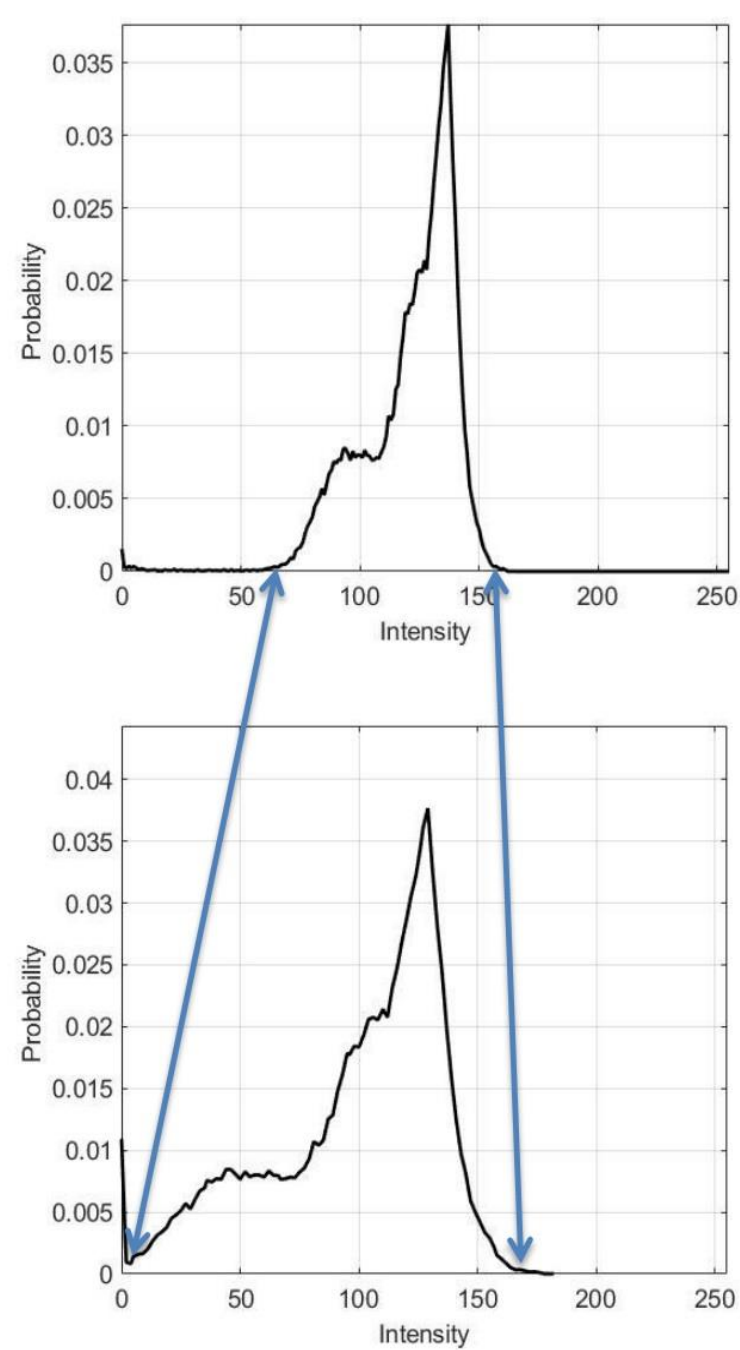

Figure. 1 Increased contrast and brightness due to the histogram extension using the membership function.

The above equation applies to each component red, green and blue individually

$\mu_{\mathrm{n}}$ is called the crossover point.

Fig. (1) shows the histogram distributions after using the membership function, where we notice the increased distribution values and it has more intensity levels, which indicates increased contrast Another fuzzy set is produced by recursion calling as follows [15]

$$
\begin{gathered}
\mu_{n}{ }^{\prime}=f_{r}\left(\mu_{n}\right)=f_{r}\left(f_{r-1}\left(\mu_{n}\right)\right), \\
r=1,2,3, \ldots,
\end{gathered}
$$

Where $f_{r}\left(\mu_{n}\right)$ being the successive applications are given by [16]:

$$
\mu_{n}^{\prime}= \begin{cases}2 \mu_{n}^{2} & 0 \leq \mu_{n} \leq 0.5 \\ 1-2\left(1-\mu_{n}\right)^{2} & 0.5 \leq \mu_{n} \leq 1\end{cases}
$$

Finally, defuzzification operation for the contrastenhanced image is calculated by:

$$
c_{\mathrm{n}}{ }^{\prime}=g^{-1}\left(\mu_{\mathrm{n}}\right)
$$

This done by:

$$
c_{\mathrm{n}}^{\prime}=\mu_{\mathrm{n}}^{\prime}\left(\mu_{\mathrm{n}_{\text {max }, \mathrm{n}}}^{\prime}-\mu_{\mathrm{n}_{\min , \mathrm{n}}}^{\prime}\right)-\mu_{\mathrm{n}_{\min , \mathrm{n}}}^{\prime}(7)
$$

$\mu_{n_{\text {min, }}}^{\prime}$ and $\mu_{n_{\text {max, }}}^{\prime}$ in inverse space of each component RGB in the image are given by:

$$
\begin{gathered}
\mu_{n \text { max }, n}^{\prime}=\mu_{i a v, n}^{\prime}+\mathrm{t} \mu_{n s t d, n}^{\prime} \\
\mu_{n \text { min, } \mathrm{n}}^{\prime}=\mu_{n a v, \mathrm{n}}^{\prime}-\mathrm{t} \mu_{n \text { std,n }}^{\prime}
\end{gathered}
$$

Where $\mu_{n \text { av,n }}^{\prime}$ and $\mu_{n \text { std,n }}^{\prime}$ the mean value and standard deviation for $\mu_{n}^{\prime}$. Fig. 2 shows the original image, the image after applying mapping in membership function and the enhanced image, and histogram distribution of the red, green and blue components in each stage. Where we notice an increase in contrast in the distribution after applying of the membership function and increase it more after the defuzzification. The steps of the proposed algorithm are given by:

a. Read color image $c(x, y, n), n=r, g \& b$

b. Extract $R, G \& B$ components.

c. Fuzzification $R, G \& B$ component by using Membership function Eq. (3).

d. Defuzzification $R, G \& B$ component by using Eq. (7).

e. Composed $R, G \& B$ component to get enhanced images.

We can shorten the proposed algorithm by using the following steps using the steps shown in Fig. 3.

\section{Quality assessment}

Several quality measures measure image quality without a reference. One of these important methods is entropy.

This scale determined by the maximum amount of information in the medical image. The entropy defined as [17]:

$$
\text { Entropy }=-\sum_{i} p(i) \log (p(i))
$$

where $p(i)$ being the probability of grey images.

Another method used to measure the quality of medical images is Wavelet Quality Evaluator (WQE) [17]. This measure relies on the wavelet transformation. 


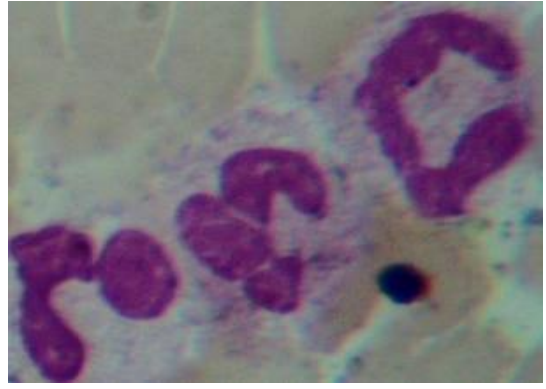

(a)

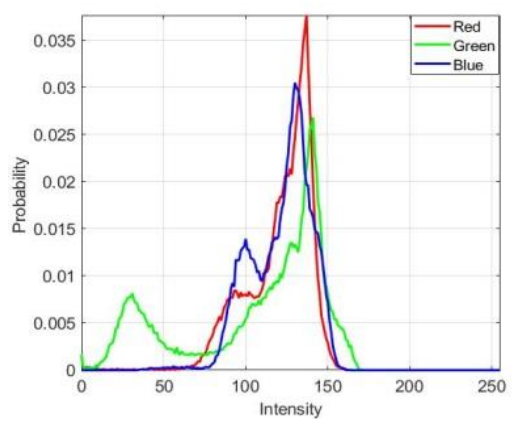

(d)

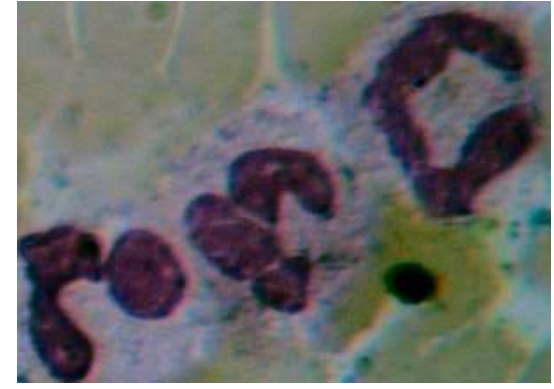

(b)

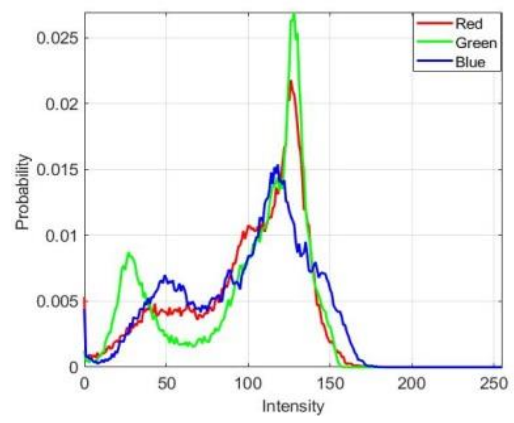

(e)

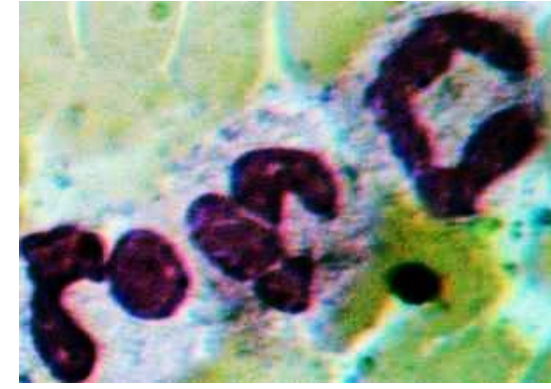

(c)

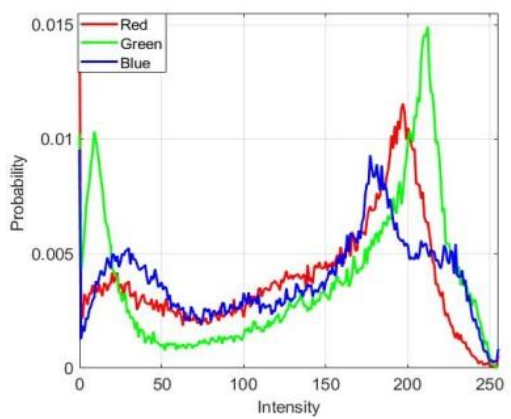

(f)

Figure. 2: (a) Original microscope image, (b) image after applying of the membership function and the final enhancement in (c), and its histogram distributions in (d), (e) and (f).

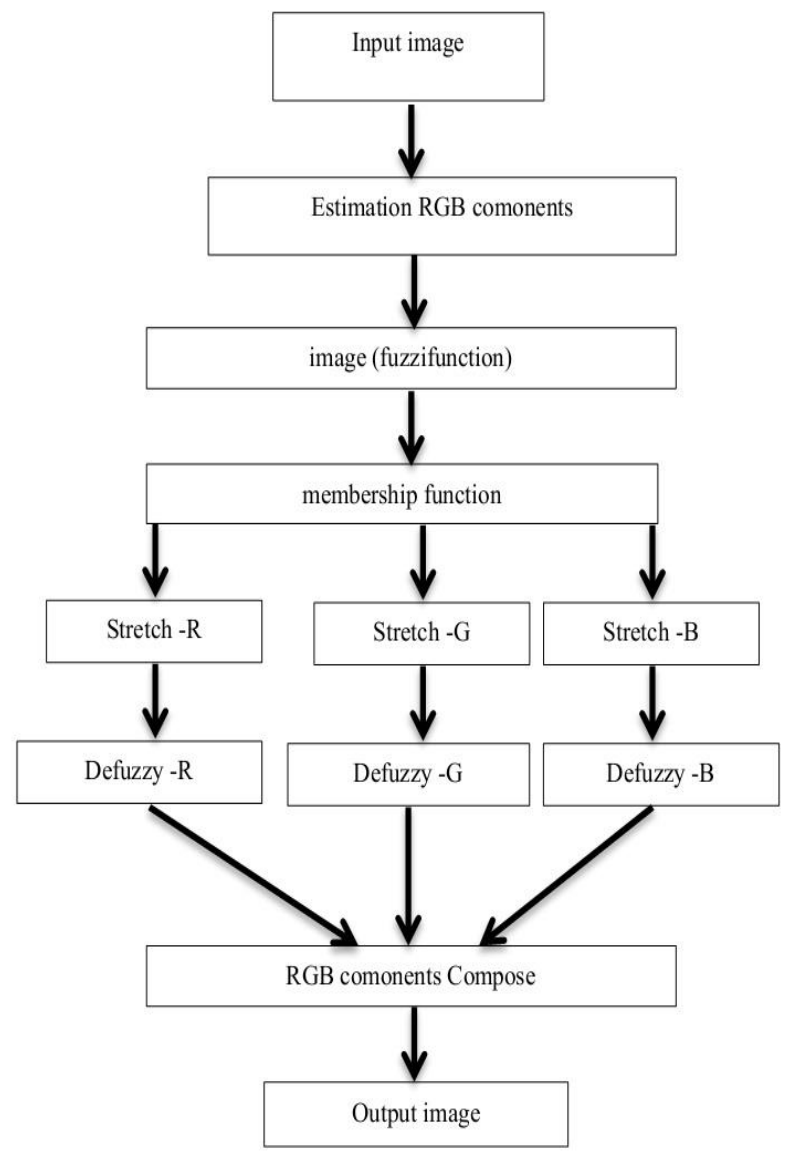

Figure. 3 Overall process of the proposed algorithm
First, the lighting component is calculated by using:

$$
v=\max (r, g, b)
$$

Then the wavelet mapping of $v$ component is found. Finally, the wavelet quality evaluator is [18]:

$$
W Q E=1 / \operatorname{std}(H L)
$$

In both scales, increasing values indicates increased quality. Also was used a Lightness Order Error (LOE) that depending on the relationship between the lightness component in the original image and the enhanced image, this scale measure naturalness. The lightness $v$ as in (Eq. 8), the difference between of the original lightness image and its enhanced lightness value ve can be written as [19]:

$$
\begin{aligned}
\operatorname{dif}(x, y) & =\sum_{i=1}^{m} \sum_{j=1}^{n}\left(U_{n}(v(x, y), v(i, j))\right. \\
\oplus U_{n}\left(v_{e}(x, y), v_{e}(i, j)\right) & \\
\mathrm{U}_{n}(\mathrm{x}, \mathrm{y}) & =\left\{\begin{array}{cl}
1, & \text { for } x \geq y \\
0, & \text { else }
\end{array}\right.
\end{aligned}
$$




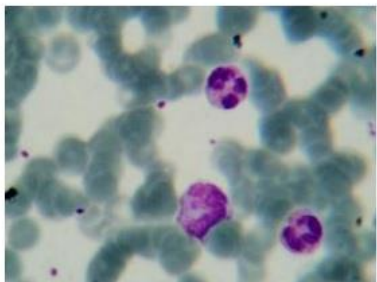

(Image 1)

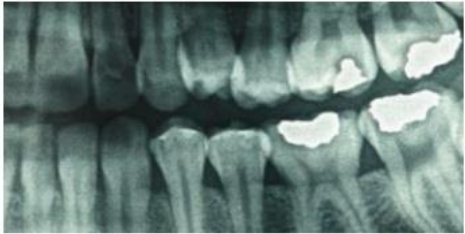

(Image 2)

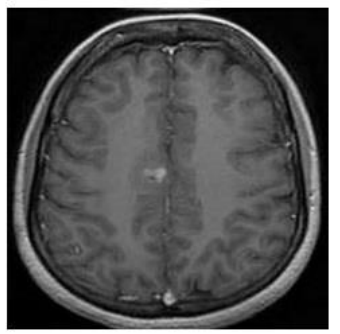

(Image 3)

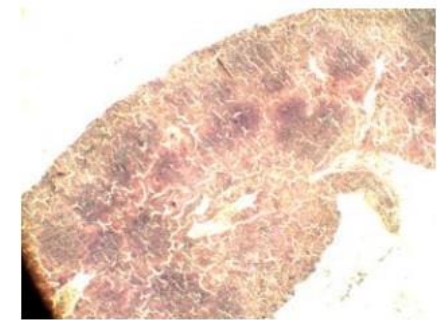

(Image 4)

Figure. 4 Original medical images used in this study

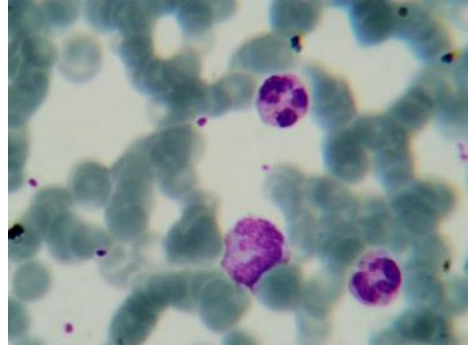

(a)

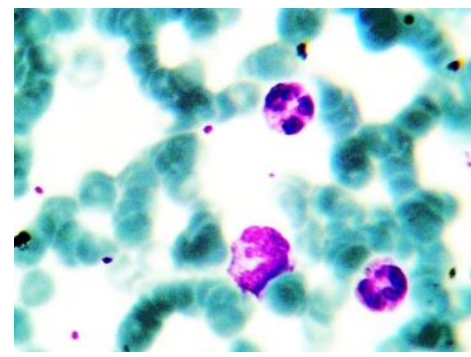

(d)

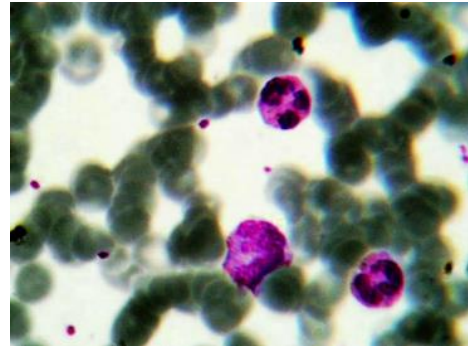

(b)

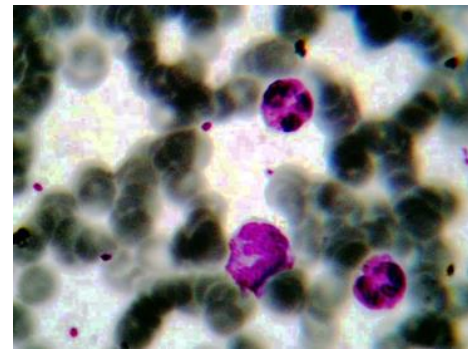

(e)

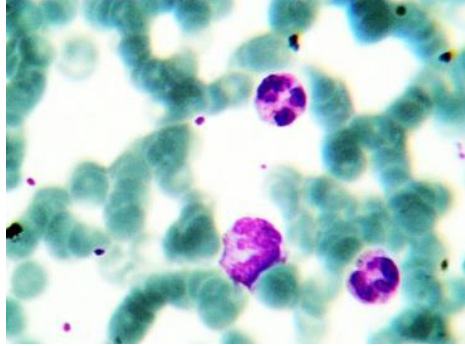

(c)

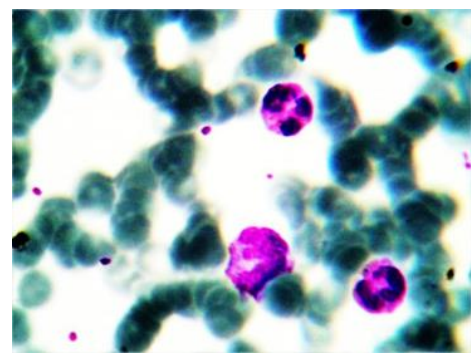

(f)

Figure. 5 Image_1 enhncement in: (a) original, (b) FLSMF, (c) FLFMS, (d) FLFMC, (e) HE, and (f) FLBGC.

Where $\mathrm{m}$ and $\mathrm{n}$ are the size of the image, $U_{n}(x, y)$ is the unit step function, $\oplus$ is the convolution operator. The LOE can be calculated as [19]:

$$
\mathrm{LOE}=\frac{1}{m * n} \sum_{i=1}^{m} \sum_{j=1}^{n} \operatorname{dif}(i, j) .
$$

In this scale, a lower value indicates good lightness improvement in the enhanced image.

\section{Experimental result}

In this research a collection of medical images have been improved by using Fuzzy logic depending on stretch membership function, three types of medical images have been improved as illustrated in Fig. 4, the first image (image1.jpg) is microscope blood cells image with size $(648 \times 486)$, the second image (image2.jpg) is dental $x$-ray image with size $(580 \times 290)$, third image (image3.jpg) magnetic resonance images with size $(256 \times 256)$ and forth image (image4.bmp) is microscope colons image with size $(648 \times 486)$. All of these medical images were processed using the MATLAB software. The suggested method FLSMF used in enhanced medical images was compared with another method (FLFMS, FLFMC, HE and FLBGC). In order to find the best way to improve, the entropy value and $\mathrm{WQE}$ are calculated. Figs. 5-8) illustrates all images enhanced by using the five methods. We note through subjective evaluation that the best method of enhancement was the proposed method, where it succeeded in improving the brightness and contrast for all types of images (microscopic, $\mathrm{x}$-ray and magnetic resonance) followed by the FLFMS then FLBGC and FLFMC, while the HE was less enhancement. This result also matches to Table 1 that shows Entropy, WQE and the LOE of them; we note that the highest values for Entropy and WQE scales were for the proposed method FLSMF, which indicates the increased contrast and clarity in those images more than the rest of the enhanced images. In the same table, we note that the few values are in LOE, it was mostly for the 


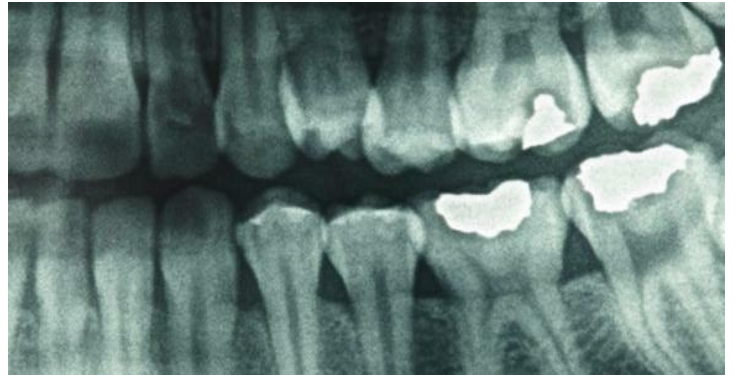

(a)

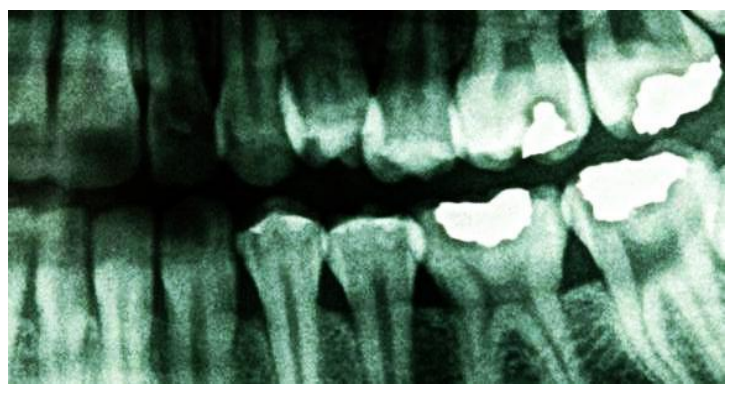

(c)

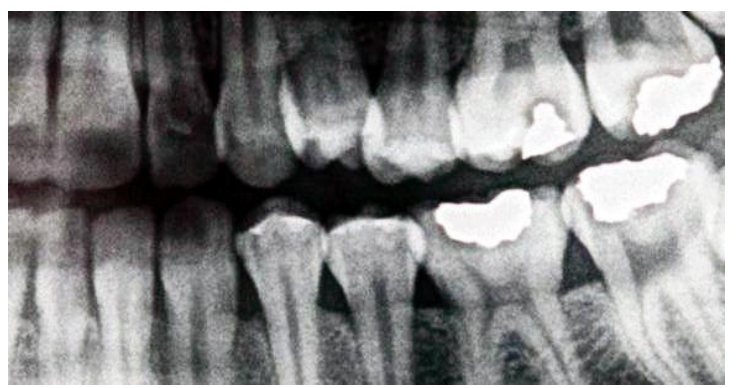

(e)

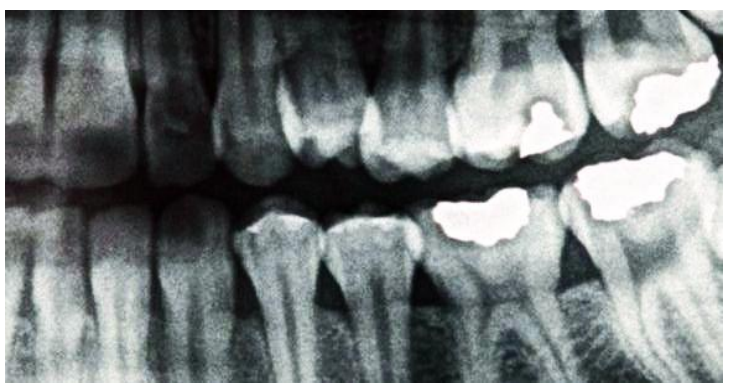

(b)

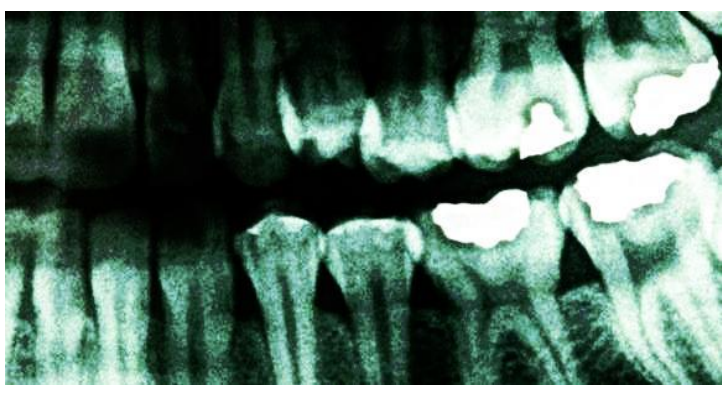

(d)

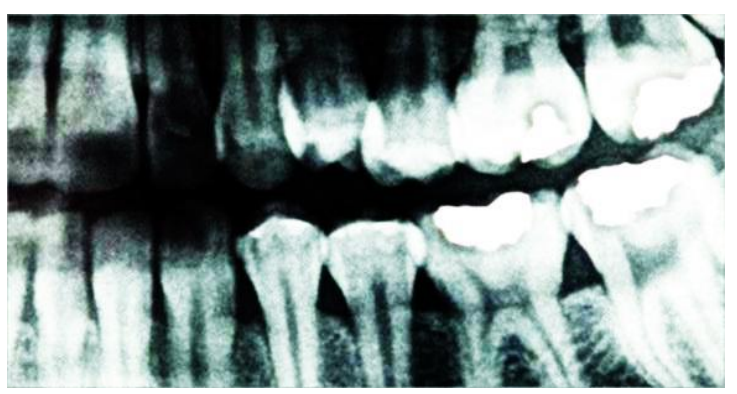

(f)

Figure. 6 Image_2 enhncement in: (a) original, (b) FLSMF, (c) FLFMS, (d) FLFMC, (e) HE, and (f) FLBGC.

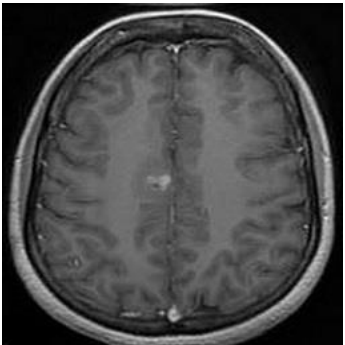

(a)

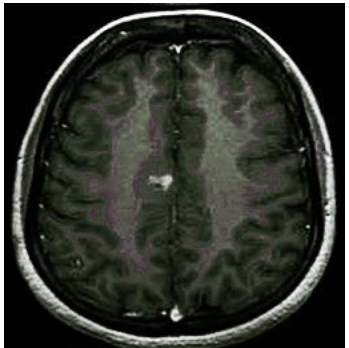

(d)

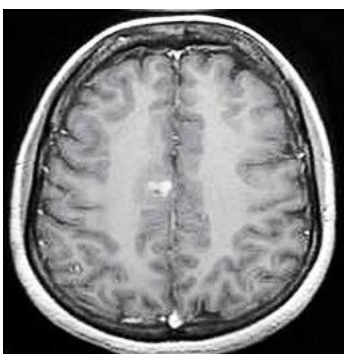

(b)

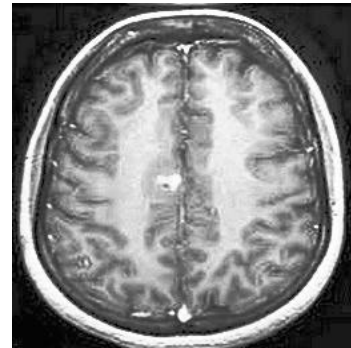

(e)

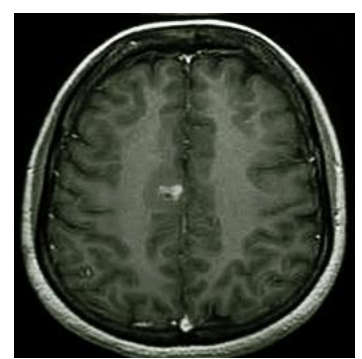

(c)

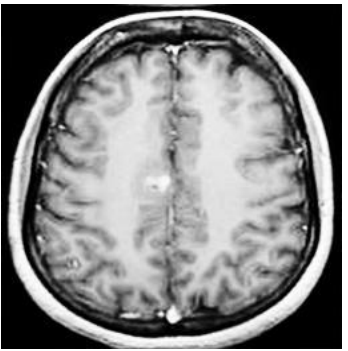

(f)

Figure. 7 Image_3 enhncement in: (a) original, (b) FLSMF, (c) FLFMS, (d) FLFMC, (e) HE, and (f) FLBGC 


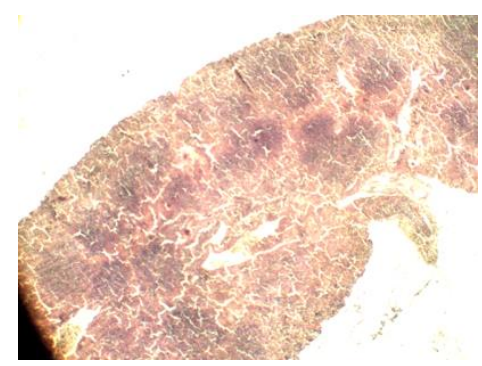

(a)

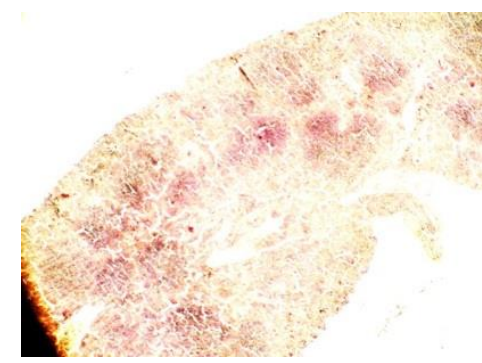

(d)

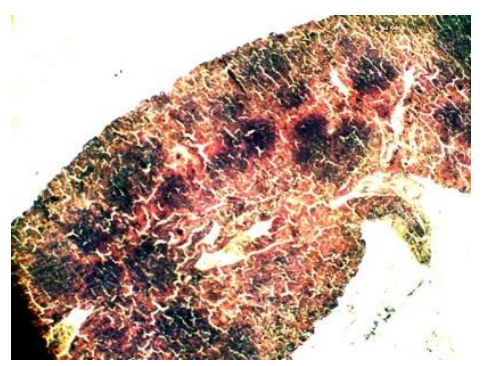

(b)

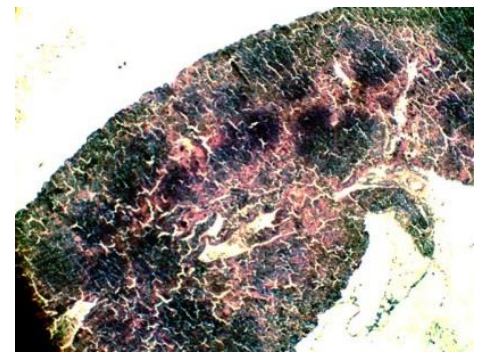

(e)

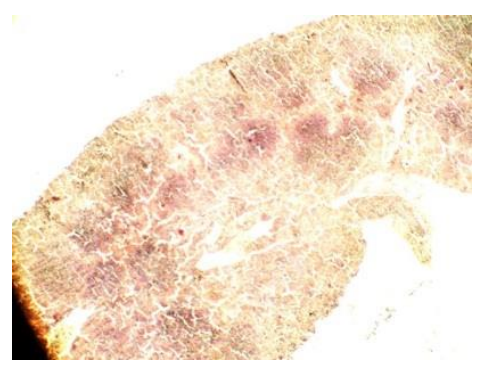

(c)

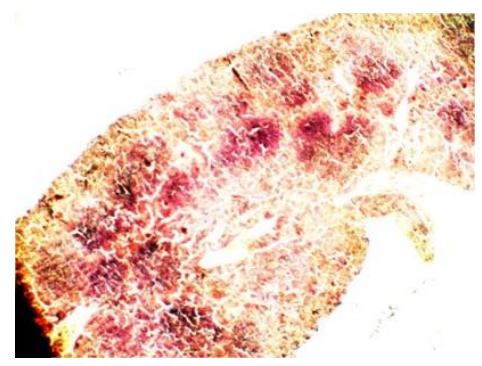

(f)

Figure. 8 Image_4 enhncement in: (a) original, (b) FLSMF, (c) FLFMS, (d) FLFMC, (e) HE, and (f) FLBGC.

Table 1. Entropy, WQE and average of them for the enhancement of the medical image

\begin{tabular}{|c|c|c|c|c|c|c|}
\hline \multirow{2}{*}{$\begin{array}{c}\text { Enhancement } \\
\text { Method }\end{array}$} & \multicolumn{3}{|c|}{ Image 1 } & \multicolumn{3}{c|}{ Image 2 } \\
\cline { 2 - 7 } & Entropy & WQE & LOE & Entropy & WQE & LOE \\
\hline FLFMS [9] & 7.005 & 0.229 & 101.009 & 7.653 & 0.092 & 51.911 \\
\hline FLFMC [9] & 6.442 & 0.168 & 154.100 & 7.196 & 0.072 & 100.272 \\
\hline HE [6] & 5.935 & 0.164 & 101.177 & 5.993 & 0.103 & 58.871 \\
\hline FLBGC[13] & 7.168 & 0.152 & 114.739 & 7.454 & 0.084 & 152.948 \\
\hline FLSMF & 7.425 & 0.171 & 77.538 & 7.686 & 0.098 & 52.562 \\
\hline Enhancement & \multicolumn{7}{|c|}{ Image 3 } & \multicolumn{3}{|c|}{ Image 4 } \\
\cline { 2 - 7 } Method & Entropy & WQE & LOE & Entropy & WQE & LOE \\
\hline FLFMS [9] & 6.384 & 0.072 & 73.599 & 5.046 & 0.152 & 150.014 \\
\hline FLFMC [9] & 6.025 & 0.063 & 117.170 & 4.469 & 0.196 & 346.820 \\
\hline HE [6] & 5.835 & 0.054 & 77.328 & 4.536 & 0.029 & 177.084 \\
\hline FLBGC[13] & 6.965 & 0.051 & 124.344 & 5.387 & 0.097 & 205.030 \\
\hline FLSMF & 6.903 & 0.053 & 58.886 & 5.795 & 0.033 & 54.128 \\
\hline
\end{tabular}

proposed method, this indicates an improvement in the goodness of the contrast while maintaining the lightness.

\section{Conclusions}

In this study, medical images were enhanced using a fuzzy technique. By analyzing these results, we can conclude the proposed method FLSMF has succeeded in improving various types of medical images and it had the good high average value of entropy (6.95) and WQE (0.08) and in the same time lower average value for the $\operatorname{LOE}(60.77)$ when it compared with other algorithms FLFMS, FLFMC, $\mathrm{HE}$ and FLBGC. This indicates that the proposed algorithm FLSMF succeeded in increasing the contrast and clarity in those images.

\section{Conflicts of Interest}

The authors declare no conflict of interest.

\section{Author Contributions}

Hazim G. Daway has contributed to the design and implementation of the research by using Matlab. Rafid Abbas Ali and Asaad M. Abbas have supervised the written paper and providing the necessary data. All authors approved the final version.

\section{References}

[1] S. Hassan, H. Daway, and I. Al-Alaway, "Improving an Illumination System in the Microscopic Imaging of Nuclear Tracks Using 
Light Emitting Diode", Indian Journal of Public Health Research \& Development, Vol. 9, No. 12, pp. 1282-1287, 2018.

[2] H. Daway, I. Al-Alaway, and S. Hassan, "Reconstruction the illumination pattern of the optical microscope to improve image fidelity obtained with the CR-39 detector", In: Proc. of AIP Conf. Proc., Vol. 2144, No. 1, pp. 030006, 2019.

[3] H. kareem, H. Daway, and E. Daway, "Underwater Image Enhancement using Colour Restoration based on YCbCr Colour Model", In: Proc. of IOP Conf. Series: Materials Science and Engineering, Vol. 571, No. 1, pp. 1-7, 2019.

[4] N. Mirza, H. Kareem, and H. Daway, "Low lightness enhancement using nonlinear filter based on power function", Journal of Theoretical and Applied Information Technology, Vol. 96. No 1, pp. 61-70, 2019.

[5] H. Daway, F. Mohammed, and D. Abdulabbas, "Aerial image enhancement using modified fast visibility restoration based on sigmoid function", Advances in Natural and Applied Sciences, Vol. 10, No. 11, pp. 16-22, 2016.

[6] Z. AMEER, H. Daway, and H. Kareem, "Enhancement underwater image using histogram equalization based on color restoration", Journal of Engineering and Applied Sciences, Vol. 14, No. 2, pp. 641-647, 2019.

[7] Z. Vance and G. James, "Luminance-contrast mechanisms in humans: visual evoked potentials and a nonlinear model", Vision Research, Vol. 46, No. 24, pp. 4163-4180, 2006.

[8] H. Daway, E. Daway, and H. Kareem, "Colour Image Enhancement by Fuzzy Logic Based on Sigmoid Membership Function", International Journal of Intelligent Engineering and Systems, Vol. 13, No. 5, pp. 238-246, 2020.

[9] J. Chang and Y. Bo, "A novel nonlinear algorithm for typhoon cloud image enhancement", International Journal of Automation and Computing, Vol. 8, No. 2, pp. 161-169, 2011.

[10] M. SJ and M. Rajeswari, "Membership Function modification for Image Enhancement using fuzzy logic", International Journal of Emerging Trends \& Technology in Computer Science (IJETTCS), Vol. 2, No. 2, 2013.

[11] G. Raju and M. Nair, "A fast and efficient color image enhancement method based on fuzzylogic and histogram", AEU-International Journal of Electronics and Communications, Vol. 68, No. 3, pp. 237-243, 2014.
[12] J. Kaur and A. Kaur, "Image Contrast Enhancement method based on Fuzzy Logic and Histogram Equalization", International Research Journal of Engineering and Technology, Vol. 3 No. 5, 2016.

[13] A. Nandal and H. Rosales, "Enhanced image fusion using directional Contrast rules in fuzzy transform domain", Springer Plus, Vol. 5, No. 1, pp. 1846, 2016.

[14] D. Mikhov, Y. Kondratenko, G. Kondratenko, and I. Sidenko, "Fuzzy Logic Approach to Improving the Digital Images Contrast", In: Proc. of the IEEE 2nd Ukraine Conf. on Electrical and Computer Engineering pp. 1183$1188,2019$.

[15] T. Ross, Fuzzy logic with Engineering Applications, John Wiley \& Sons, 2005.

[16] F. Zhou, Z. Jia, J. Yang, and N. Kasabov, "Method of improved fuzzy contrast combined adaptive threshold in NSCT for medical image enhancement", BioMed Research International, Vol. 2017, 2017.

[17] Rafael C and Richard E, Digital Image Processing, Second Edition, Prentice Hall, 2002.

[18] S. Ahmed, H. Daway, and H. Rashid, "Quality of medical microscope Image at different lighting condition", In: Proc. of IOP Conf. Series: Materials Science and Engineering, Vol. 871, No. 1, pp. 012072, 2020.

[19] S. Wang, J. Zheng, H. Hu, and B. Li, "Naturalness Preserved Enhancement Algorithm for Non-Uniform Illumination Images", IEEE Transactions on Image Processing, Vol. 22, No. 9, pp. 3538-3548, 2013. 